Special Issue: Russian Fracture Mechanics School

\title{
Fractal statistics of brittle fragmentation
}

\author{
M. Davydova, S. Uvarov \\ Institute of Continuous Media Mechanics Ural Branch Russian Academy of Sciences, 1, Ac. Korolev str., 614013 Perm, Russia. \\ davydova@icmm.ru; usv@icmm.ru
}

\begin{abstract}
The study of fragmentation statistics of brittle materials that includes four types of experiments is presented. Data processing of the fragmentation of glass plates under quasi-static loading and the fragmentation of quartz cylindrical rods under dynamic loading shows that the size distribution of fragments (spatial quantity) is fractal and can be described by a power law. The original experimental technique allows us to measure, apart from the spatial quantity, the temporal quantity - the size of time interval between the impulses of the light reflected from the newly created surfaces. The analysis of distributions of spatial (fragment size) and temporal (time interval) quantities provides evidence of obeying scaling laws, which suggests the possibility of selforganized criticality in fragmentation.
\end{abstract}

KEYWORDS. Fragmentation of brittle materials; Fractal statistics; Self-organized criticality.

\section{INTRODUCTION}

$\mathrm{F}$ ragmentation is the process of breaking a solid into separate fragments caused by multiple fractures. Such phenomenon can be observed in both engineering and natural objects over a wide range of spatial and temporal scales. An investigation of fragmentation statistics generally includes the determination of the cumulative distribution of fragment sizes or masses, i.e., the number of fragments $N(m)$ with a size or mass larger than $S$ or $m$, respectively. The distribution type depends on loading conditions, material characteristics and sample geometries. Many types of distribution functions have been observed experimentally: log-normal, power-law, Mott, exponential, Weibull, and combined exponential and power-law [1 - 8]. Summarizing the results of experimental data processing, we can classify all these distribution functions into two groups: exponential and power law. The assumption that the exponential distribution is typical of the fragmentation of ductile materials and the power-law distribution characterizes brittle fragmentation has been discussed by Grady [7]. Donald Turcotte [8] has pursued the fragmentation of brittle materials as a fractal process resulting in the power law distribution function $N \sim x^{-d}$, where $N$ is the number of fragments, $x$ is the linear dimension of fragments, and $d$ is the fractal dimension. The fractal character of the distribution function in a wide range of fragment sizes allows Oddershede et al. [3] to suppose that the fragmentation exhibits self-organized criticality (SOC). In their seminal paper Per Bak, Chao Tang and Kurt Wiesenfeld [9] present a new concept of SOC; numerical simulation allows them to describe the behavior of a sand pile (example of a self-organized critical system) and to conclude:

$\checkmark$ distribution of life times of avalanches obeys a power law;

$\checkmark$ distribution of avalanche sizes follows a power law as well.

In other words, to prove that the system exhibits SOC, it is necessary to establish the existence of a power law for temporal and spatial quantities. The purpose of this experimental study is to demonstrate that the fragmentation process exhibits SOC. To this end, we need to determine the distribution of temporal and spatial variables. It has been shown that for the fragmentation of glass plates under quasi-static loading there exists scaling in the crack pattern formed during fragmentation and fragment size distribution are also described by the power law. The experiments on quartz cylindrical 
rods under dynamic loading were carried out to test the effect of sample size and variation in loading type. It was found out that the type of distribution function did not change, it remained a power law. The fragment size distribution was determined using a standard sieve analysis technique and electronic balance. In order to investigate temporal scaling, an original method was proposed. The main idea was to measure the kinetic of the appearance of the fracture surface. For a loaded glass sample illuminated with the light source, the newly formed fracture surface produced light impulses, which were registered by Photo Multiplayer Tube connected to the oscilloscope. The temporal variable was the interval between impulses, or in other words, the time between the fracture events. The statistical data processing shows that the distribution of time intervals obeys the power law. In the case of analyzing data corresponding to the initial time of the process the distribution of time interval is governed by the exponential law. The explanation of the fact that fracture kinetics at the initial stage is characterized by another distribution function requires additional investigation.

\section{FRAGMENTATION OF THE GLASS PLATES UNDERQUASI-STATIC LOADING}

$\mathrm{Q}$ uasi-static testing was performed in the experiments with glass plates loaded in a "sandwich" to save the glass fragmentation pictures (Fig. 1a). Using the original software, the images were transformed into schematic pictures corresponding to the fragmentation patterns (Fig. 1b). This allowed us to determine the size and number of fragments and the total length of cracks. We consider two types of scaling. The first type is based on the relation

$$
L(r) \sim r^{D}
$$

where $L(r)$ is the total crack length in the boxes of a size $r \times r$ centred at the point $B$ (Fig. 1b), and $D$ is the fractal dimension. The second type is the traditional definition of the cumulative distribution of fragment sizes, in other words, the calculation of the number of fragments $N(S)$ with a size larger than $S$.

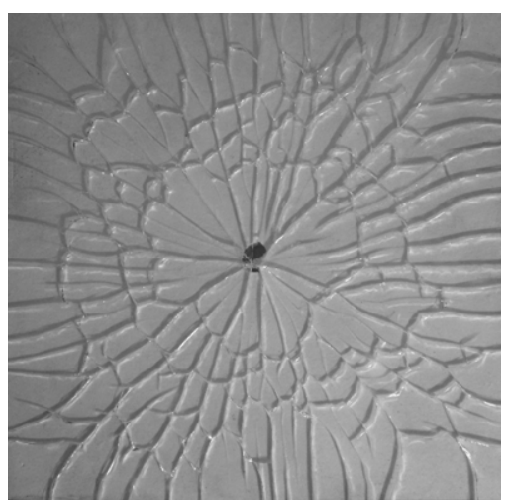

(a)

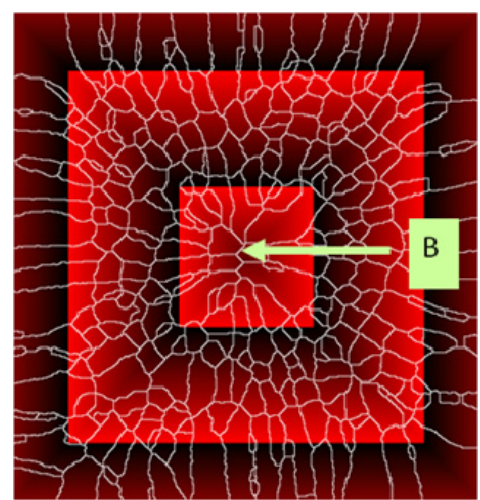

(b)

Figure 1: a) Photo of typical fragmentation patterns. b) Schematic pictures of the fragmentation patterns used to determine the size and number of fragments and the total length of cracks.

The use of expression (1) was discussed by Sornette et al. [10]. However, it should be noted that the fracture pattern [10] does not have a distinct central point. At the same time, the examined fragmentation patterns have a central point, and their configuration is similar to that created with the model of diffusion-limited aggregation (DLA) [11] or the model of dielectric breakdown (DB) [12]. Relation (1) can be used to define the fractal dimension for both these models. In the case of the DB model, $L(r)$ is the total length of the discharge branches within the circle of radius $r$ For the DLA model, $L(r)$ is the number of particles. By analogy with the DLA and DB models, to determine the fractal dimension of the fragmentation patterns, we use relation (1), where $L(r)$ is the total length of cracks in the boxes of a size $r \times r$ centred at the point $B$ (Fig. 1b). The minimal number of the boxes used for calculation of the fractal dimension is 200 . The scaling law obtained using the relation for the crack length (1) is presented in Fig. 2a. The processing of the fragment sizes shows that the relation between the fragment area and number is also fitted by a power law (Fig. 2b). 


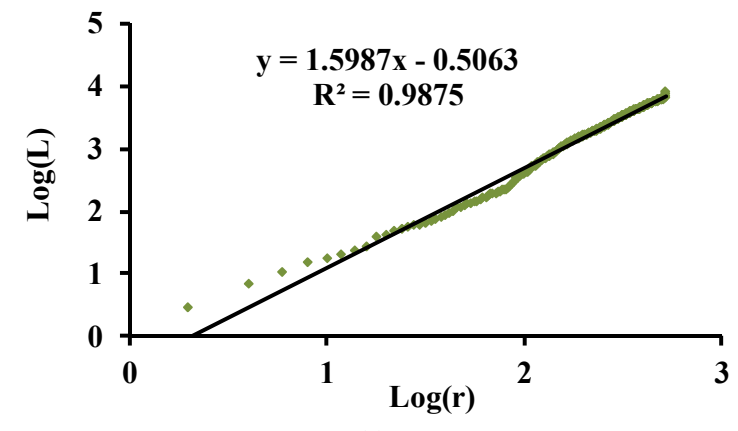

(a)

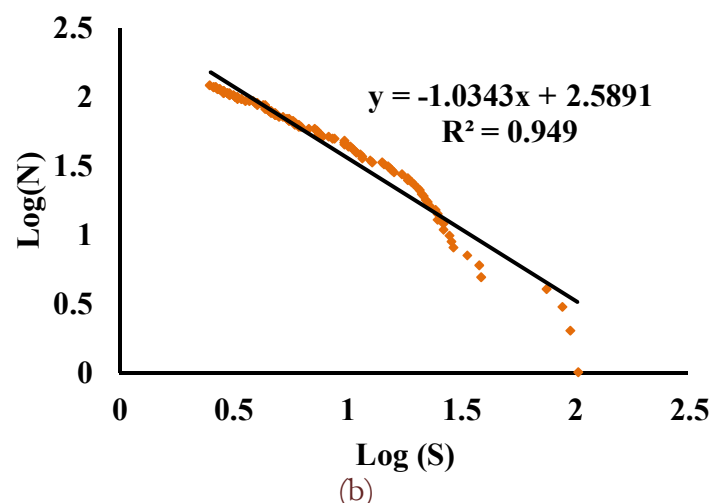

(b)

Figure 2: a) Scaling law obtained using the relation for crack length (1). b) Cumulative distribution of the fragment size in a log-log plot.

There are two types of energy-dependent fragmentation patterns leading to a breakup (Fig. 3). Fig. 3a presents a fragmentation pattern that illustrates the fracture accompanied by radial crack formation. The right fragmentation pattern (Fig. 3b) has two zones corresponding to two different fracture mechanisms. The central zone has a radial crack only. In the second zone, the crack branching process is observed. Suppose that different fracture mechanisms characterize different fractal dimensions, we have

$$
L(S) \sim S^{D}
$$

where $L(S)$ is the total crack length inside the square frame with a thickness $h$ (Fig. 3a), $S$ is the frame area, and $D$ is the fractal dimension. Calculation the fractal dimension using expression (2) shows that for the left fragmentation pattern (Fig. 3a) the data is fitted by a single line, whereas for the right pattern the log-log representation of $L(S)$ changes a slope, and the power-law exponent $D$ decreases. A change in the fracture mechanism (from radial crack-to-crack branching) correlates with the qualitative changes in the fractal dimension.
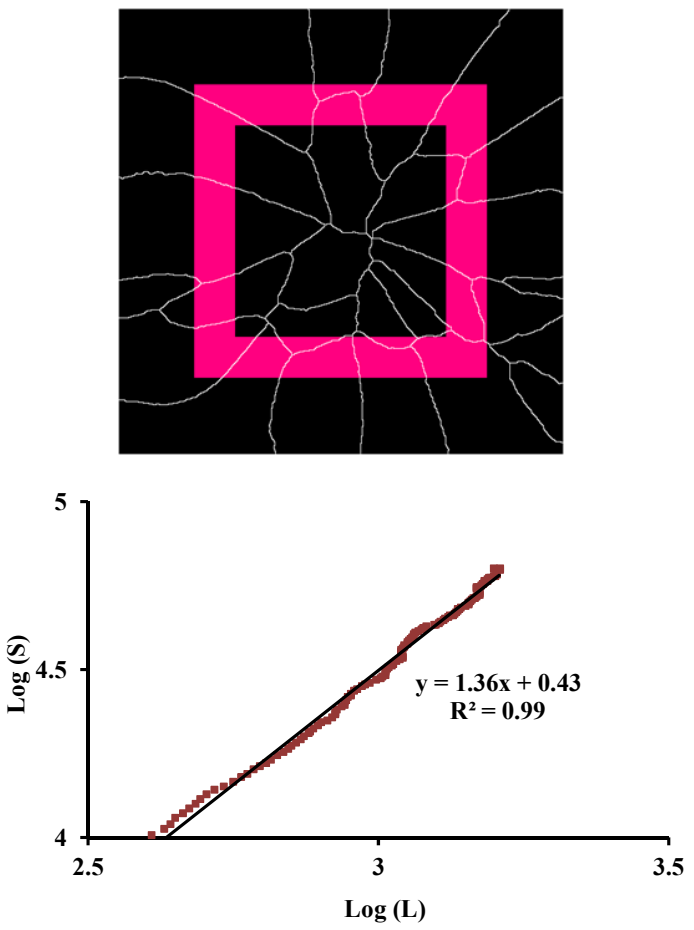

(a)
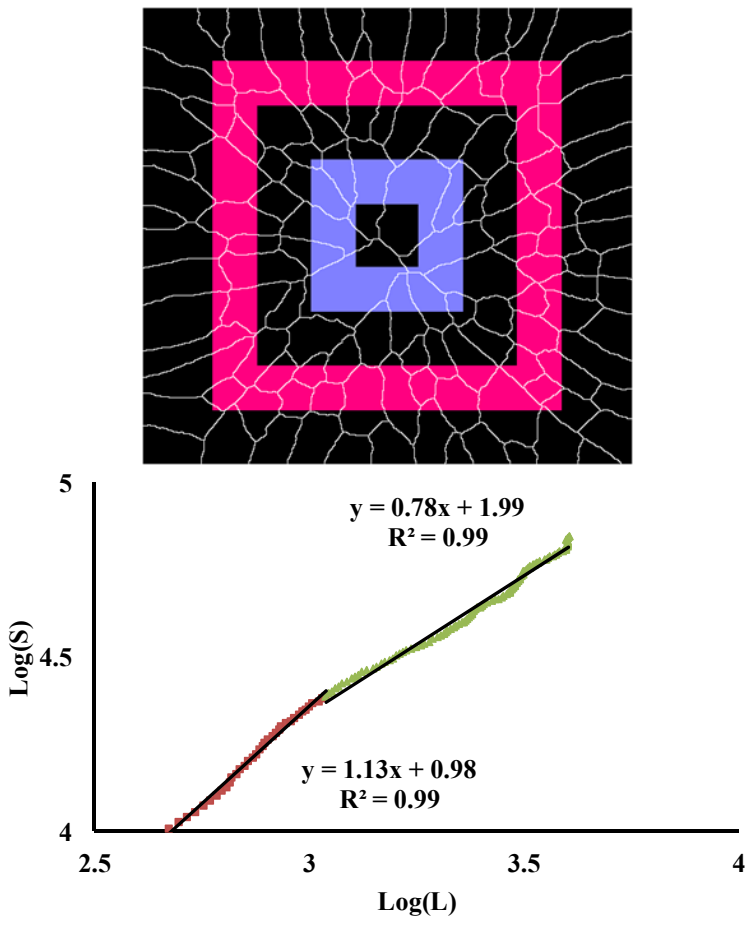

(b)

Figure 3: a) Fragmentation pattern and fractal dimension definition for the fracture accompanied by radial crack formation. b) Fragmentation pattern and fractal dimension definition for the fracture accompanied by a change in the fracture mechanism. 


\section{FRAGMENTATION OF QUARTZ RODS UNDER DYNAMIC LOADING}

\section{Spatial Scaling}

7 he fragmentation statistics was studied in recovery dynamic experiments with loaded quartz cylindrical rods using a ballistic set-up, which consisted of a gas gun with bore diameter of $19.3 \mathrm{~mm}$, a velocity registration system and a base where the specimen was placed (Fig. 4). The sectional glass rod was composed of a buffer and the main part covered by an elastic shell. The buffer was used for realization of uniaxial loading produced by a cylindrical projectile of mass $13.9 \mathrm{~g}$ accelerated up to the velocities of $6-20 \mathrm{~m} / \mathrm{s}$. The mass of the fragments passing through the sieves was obtained by weighting the fragments using an electronic balance HR-202i (accuracy $10^{-4} \mathrm{~g}$ ). The mass of the fragments corresponding to the maximum of the probability density function $m_{\max }$ varied in the range from $2 * 10^{-4} \mathrm{~g}$ to $6^{*} 10^{-4} \mathrm{~g}$ (Fig. $5 a)$. The cumulative fragment size distribution, i.e. the number of fragments $N(m)$ with a mass greater than a specified value $m$, was fitted by the power law (Fig. 5b).

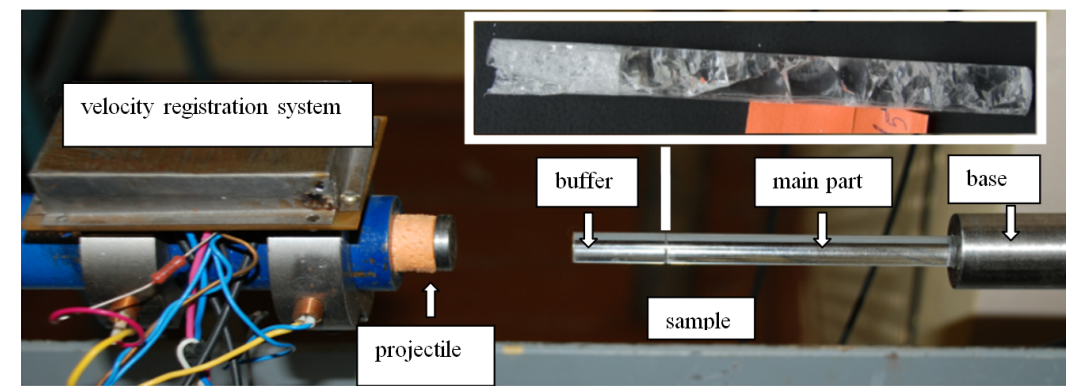

Figure 4: Ballistic set-up. An example of the fragmentation pattern is given in the upper right-hand corner.

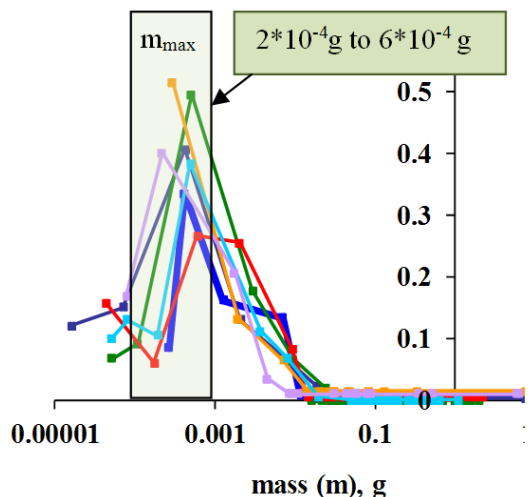

(a)

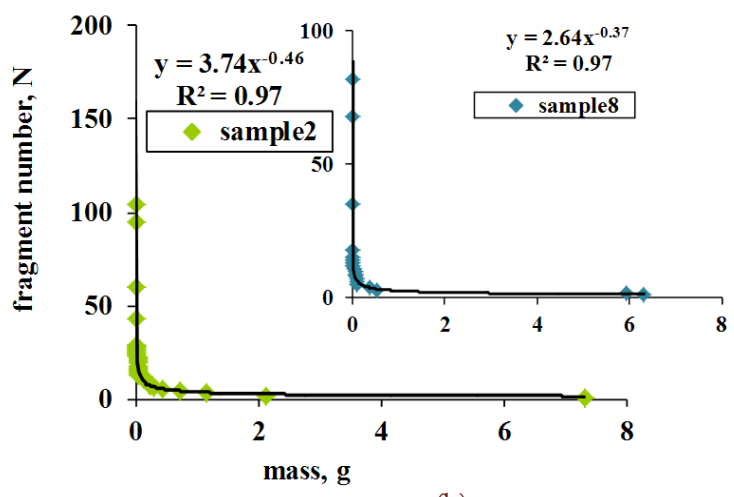

(b)

Figure 5: a) Probability density function (different colors correspond to different samples). b) Cumulative fragment size distribution.

In order to avoid the possible influence of the reflected wave on the fragmentation scenario, the ballistic set-up was modified. The sample was placed into a steel cylinder filled with plastic foam (Fig. 6). The sectional glass rod was composed of the buffer, the main part and the outer part. The presence of the last part allowed us to catch the reflected wave.

The fragmentation statistics was analyzed by varying the sample size and load intensity (projectile velocity). The results of experiments have indicated that the variation in the sample size and loading conditions does not lead to the change in the type of probability density and cumulative mass distribution functions. We have analyzed the dependence of $m_{\max }$ on the projectile energy (Fig. 7). The markers indicate the fragmentation under different loading conditions: circles correspond to the fragmentation due to interaction between the direct and reflected compression waves (Fig. 4); triangles correspond to fragmentation under the action of a compression wave (Fig. 6); boxes correspond to fragmentation induced by a direct compression wave and its reverberation in rod (Fig. 9). At low energy of the projectile, a more considerable scattering was found for $m_{\max }$. This actually means that for low energy we have two or three sieves with a comparable number of fragments, and for high energy - only one sieve with a predominant number of fragments. 


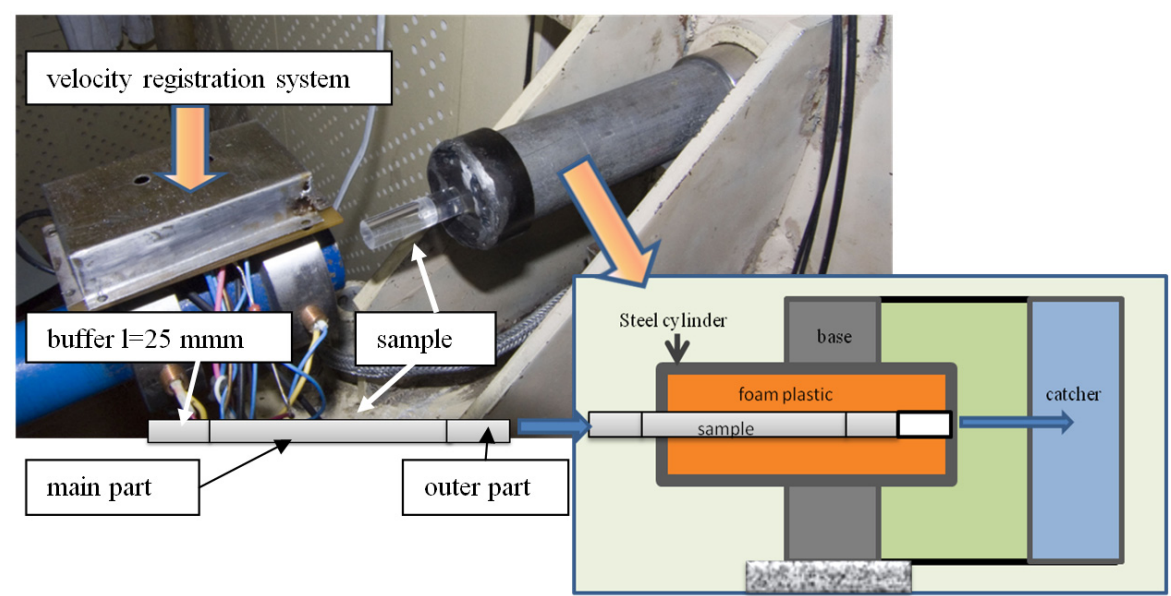

Figure 6: Ballistic set-up and scheme of steel cylinder with a sample.

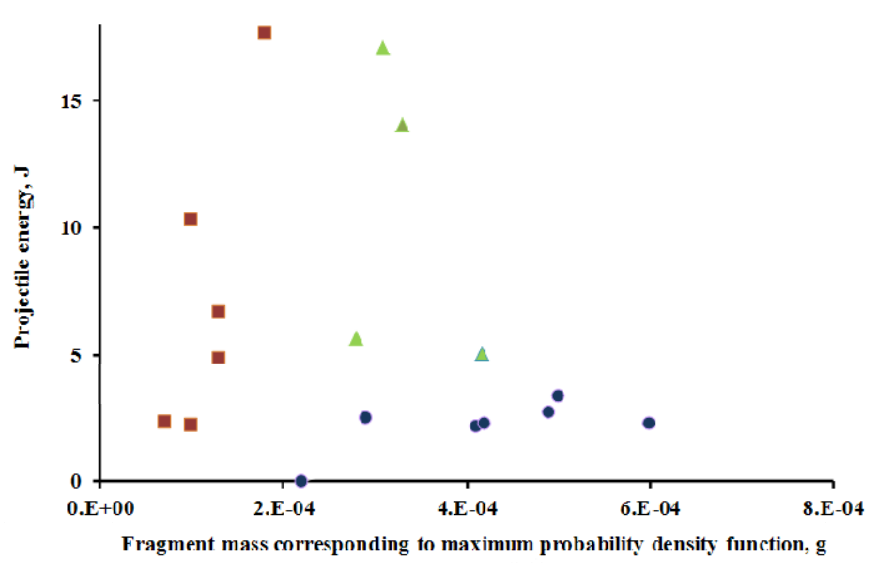

Figure 7: The dependence of fragment mass corresponding to maximum probability density function on the projectile energy.

Thus, the cumulative distributions illustrating the relation between the numbers of fragments and their linear dimension are represented as a log-log plot (Fig. 8). The linear dimension is defined as a cube root of mass or a square root of area. The distribution is fractal by nature with a power law in the form $N(>r)=C r^{-D}$, where $N$ is the number of fragments with a characteristic linear dimension greater than $r$. The fractal dimension $D$ varies from 1.6 to 2.0 for a plate and from 1.1 to 1.7 for a rod.

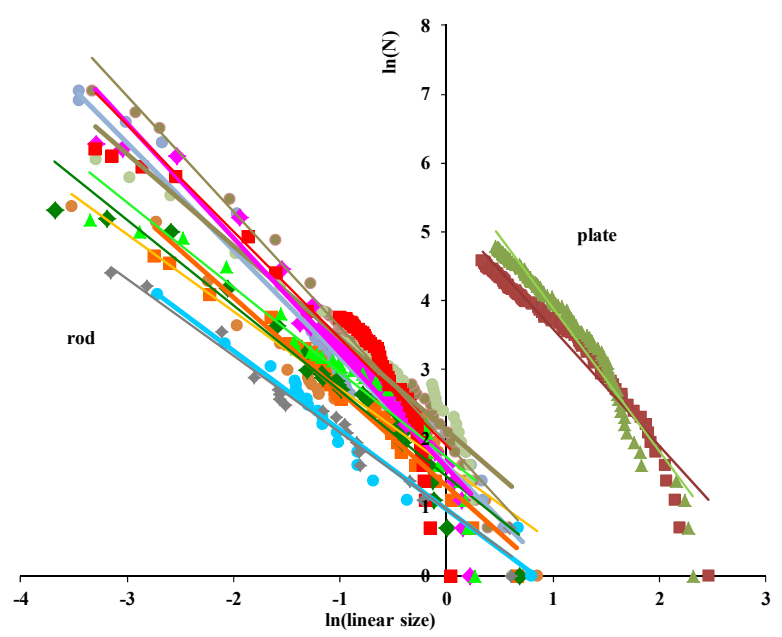

Figure 8: Double logarithmic plot of the cumulative distribution function for a plate and a rod (different colors correspond to different samples). 


\section{Temporal Scaling}

As shown in the section Introduction, to confirm the fact that the fragmentation exhibits SOC, we need to establish the existence of a power law for temporal and spatial quantities. The evidence of the spatial scaling for the fragmentation of brittle materials under different loading conditions was given in two previous sections. For measurement of temporal scale we developed an experimental device to determine the time interval between the impulses of the light reflected from the newly created fracture surfaces. In our investigation (fragmentation of quartz rods under dynamic loading), SOC means that there exist:

$\checkmark$ power law distribution of fragment size;

$\checkmark$ power law distribution of time interval between the impulses of the light reflected from newly created surfaces.

To obtain the fragment size distribution, the technique described in the previous experiments was used, which made it possible to get the distribution obeying the scaling law.

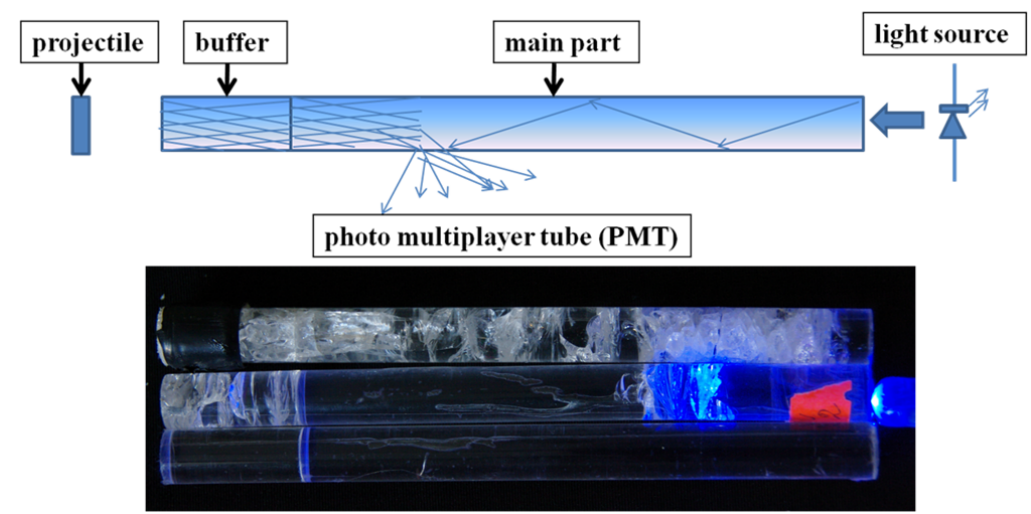

Figure 9: Scheme of the experiment for measuring the distribution time interval between the impulses of the light reflected from the fracture surfaces. The photo of the sample illuminated with the light source.

The scheme given in Fig. 9 illustrates the experimental technique used to measure the distribution of time quantities. The sample is illuminated with the light source. The intensity of the light reflected from the fracture surfaces is registered by the Photo Multiplayer Tube connected with the oscilloscope (oscilloscope sample rate is $1 \mathrm{GHz}$ ).

a)

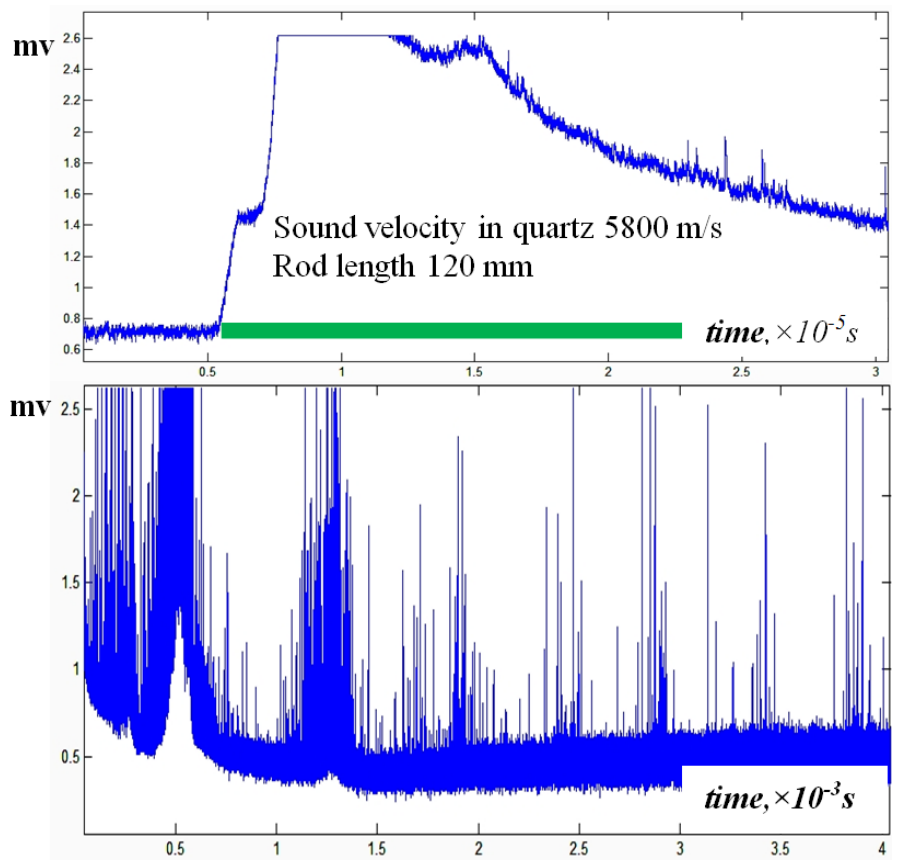

Figure 10: Signal from the oscilloscope corresponding to the intensity of the light reflected from the newly created surfaces. 
The registered signal corresponds to the change in the scattered light intensity on the interface between the fractured and unfractured parts of the sample. The appearance of the new surfaces produces the impulses with a sharp front. Fig. 10 shows the signal from the oscilloscope. The bottom plot (Fig. 10b) is the total signal, and the upper plot (Fig. 10a) corresponds to the initial stage of the process. These two plots have different time scales. The green bar indicates the propagation time of the compression wave. The time during which the signal is registered is greater $(6 \mathrm{~ms})$ than the time of compression wave propagation $(20 \mu s)$.

The first step of data processing is signal filtration. The definition of the size of time interval between the impulses of light reflected from the fracture surfaces is presented in Fig. 11. The second step involves measuring the distance between the green bars showing a sharp rise in impulse. We consider only the impulses which cross the red line. All the impulses below the red line are noise.

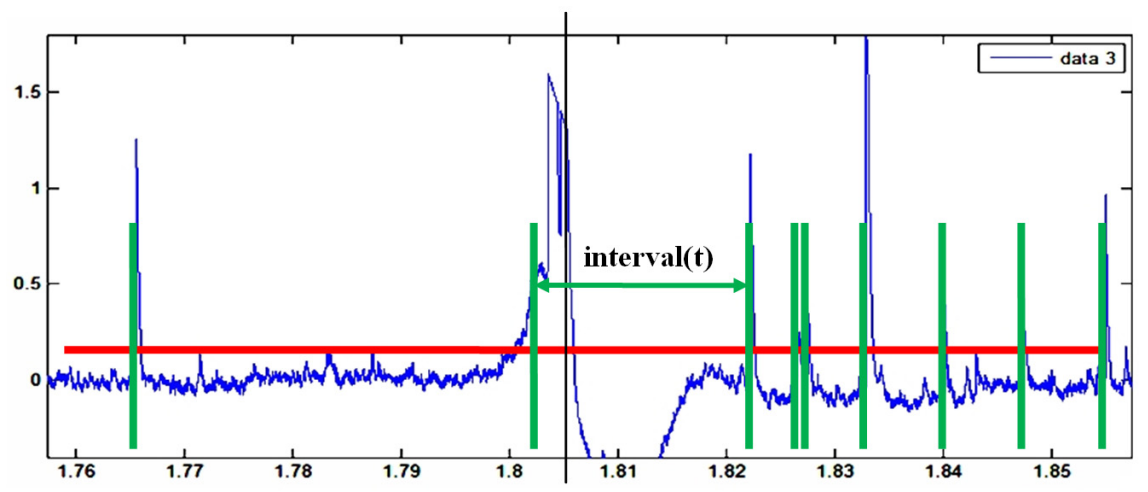

Figure 11: Determination of the size of time interval between the impulses of the light reflected from the facture surfaces.

The cumulative distribution function of the time interval in the double logarithmic plot (Fig. 12) is fitted by the straight line (total number of points 1073). At small sizes (77 points $-7.1761 \%$ of the total number of points), the curve deviates from the straight line because the size of time interval is comparable with the oscilloscope sample rate $(1 \mathrm{GHz})$. The falloff at the largest interval sizes (16 points $-1.5 \%$ of the total number of points) is due to finite-size effects. In this case the time interval is comparable with the process time. The central part is the line covering $91.3 \%$ of the total number of points.

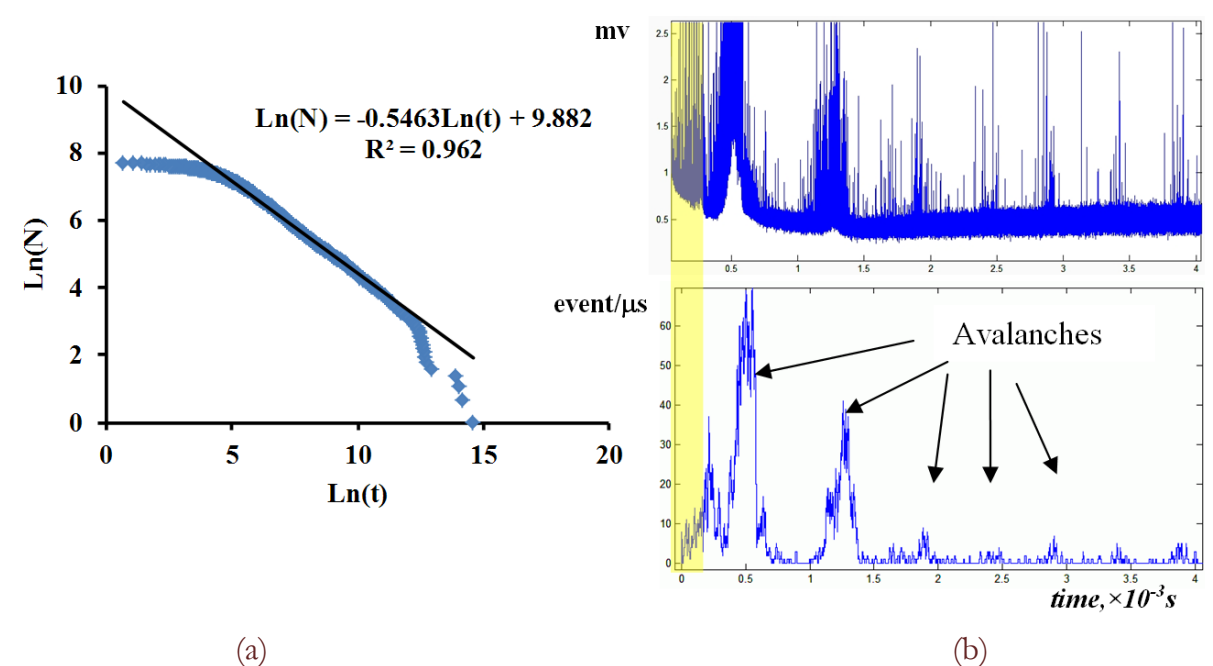

Figure 12: a) Cumulative distribution function of time interval in the double logarithmic plot. b) The signal from the oscilloscope and the frequency of impulse appearance.

The process of light reflection looks like the process of avalanche spreading (Fig. 12b). The lower plot represents the event frequency. The events are distributed in blocks. We have analyzed the time interval distribution in avalanches and found that the distribution at the initial stage (marked in yellow in Fig. 12b) cannot be described by the power law. 


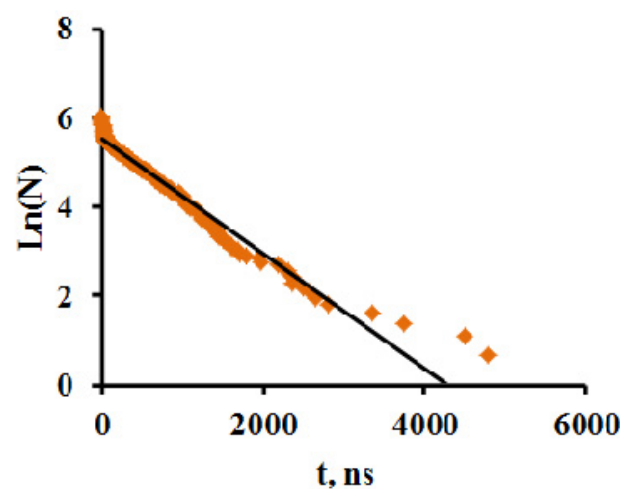

(a)

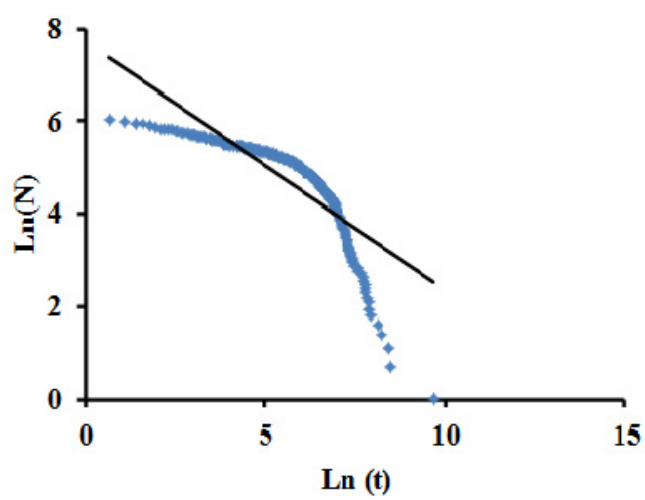

(b)

Figure 13: a) Cumulative distribution function of time interval for initial stage in a double logarithmic plot. b) Cumulative distribution function of time interval for initial stage in a coordinate system $L N(N)$-time $t$.

The left plot in Fig. 13 illustrates the distribution at the initial stage in double logarithmic coordinates. In the right plot in Fig. 13, only the vertical coordinate is logarithmic. The time interval distribution is subjected to the exponential law. An exponential functional form requires a characteristic length scale which can be defined as

$$
X_{c h}=t V
$$

where $X_{c h}$ is the characteristic size, $t$ is the x-coordinate of point C ( $\left.t=457 \mathrm{~ns}\right)$, and $V=5800 \mathrm{~m} / \mathrm{s}$ is the sound velocity in quartz. We suppose that this length scale $X_{c h}=2.6 \times 10^{-6} m$ correlates with the characteristic length scale of structure heterogeneity of quartz (Fig. 14). This problem will be the object of our future research. The initial stage statistics does not change the total statistics, because only $10 \%$ of the points belong to the initial stage.

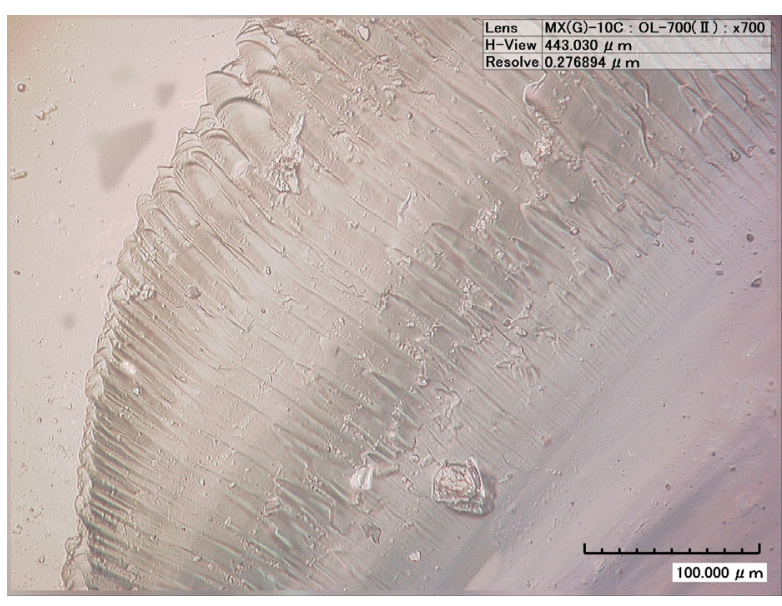

(a)

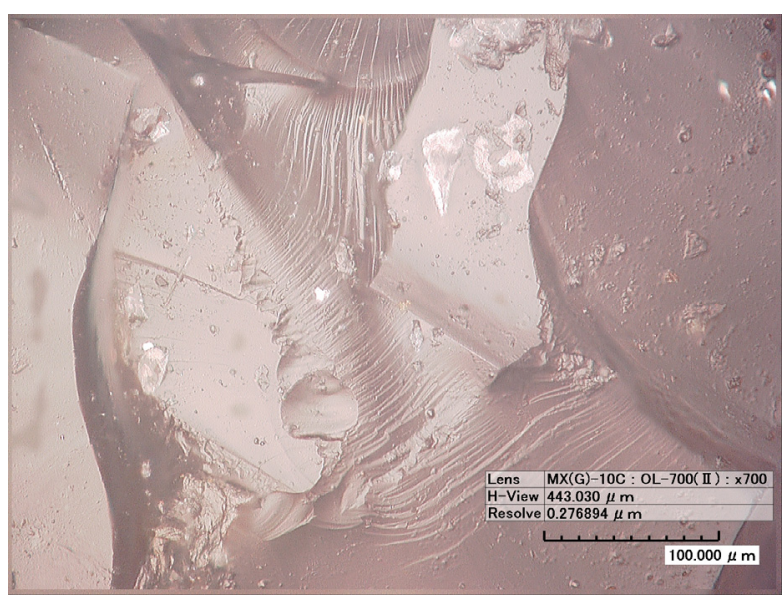

(b)

Figure 14: Fracture surface of quartz. Optical microscopy.

\section{CONCLUSION}

$\mathrm{E}$ xperimental investigations have been carried out to examine the fragmentation of brittle materials under quasistatic and dynamic loading conditions. Based on the obtained results, we can conclude:

$\checkmark$ fragmentation patterns of glass plates are fractal;

$\checkmark$ variation in the fracture mechanism of plates correlates with the changes in the fractal dimension;

$\checkmark$ fragment size distribution for the observed type of fragmentation is fractal and satisfies the relation $N(>r)=\mathrm{Cr}^{-D}$

$\checkmark$ fragment size distributions and time interval distributions show evidence of obeying scaling laws, which suggests the possibility of self-organized criticality in fragmentation. 


\section{ACKNOWLEDGMENTS}

$\mathrm{W}$

e are very grateful to Vladimir Leont'ev for help in performing experiments. The author would like to acknowledge the Russian Foundation for Basic Research (grant RFBR 11-01-96010, grant RFBR 11-01-00712).

\section{REFERENCES}

[1] D. E. Grady, M. E. Kipp, J.Appl. Phys., 58(3) (1985) 1210.

[2] T. Kadono, Phys. Rev. Letters., 78(8) (1997) 1444.

[3] L. Oddershede, P. Dimon, J. Bohr, Phys. Rev. Letters., 71(19) (1993) 3107.

[4] V. V. Sil'vestrov, Combustion, Explosion, and Shock Waves, 40(2) (2004) 225.

[5] J. Weiss, Engineering Fracture Mechanics, 68 (2001) 1975.

[6] E. S. C. Ching, S. L. Lui, X. Ke-Qing, Physica A, 287 (2000) 83.

[7] D. E. Grady, International Journal of Fracture, 153(1-2) (2010) 85.

[8] D. L. Turcotte, Fractals and chaos in geology and geophysics. Cambridge University Press, UK (1997).

[9] P. Bak, C. Tang, K. Wiesenfeld, Phys. Rev. Lett., 59(4) (1987) 381.

[10] A. Sornette, P. Davy, D. Sornette, Phys. Rev. Letters, 65(18) (1990) 2266.

[11] L. Niemeyer, L. Pietronero, H. J. Wiesmann, Phys. Rev. Letters, 52(N12) (1984) 1033.

[12] T.A. Witten, L.M. Sander, Phys. Rev. Letters, 47 (19) (1981) 1400. 